\title{
Fuzzy r-minimal Preopen Sets And Fuzzy $r-M$ Precontinuous Mappings On Fuzzy Minimal Spaces
}

\author{
민 원 근 ${ }^{*}$ 김영기 ${ }^{* *}$ \\ Won Keun Min and Young Key Kim \\ * 강원대학교 수학과 \\ ** 명지대학교 수학과 \\ 요 약
}

fuzzy r-minimal preopen set, fuzzy $\mathrm{r}-M$ precontinuous와 fuzzy $\mathrm{r}-M$ preopen 함수의 개념을 소개하며 특성들을 조사 한다.

\section{Abstract}

We introduce the concept of fuzzy $\mathrm{r}$-minimal preopen set on a fuzzy minimal space. We also introduce the concept of fuzzy $\mathrm{r}-M$ precontinuous mapping which is a generalization of fuzzy $\mathrm{r}-M$ continuous mapping, and investigate characterization of fuzzy $\mathrm{r}-M$ precontinuity.

Key Words: fuzzy minimal structures, $\mathrm{r}-$ minimal open, $\mathrm{r}-$ minimal preopen, fuzzy $\mathrm{r}-M$ continuous, fuzzy $\mathrm{r}-M$ precontinuous

\section{Intorduction}

The concept of fuzzy set was introduced by Zadeh [5]. Chang [1] defined fuzzy topological spaces using fuzzy sets. In [2], Chattopadhyay, Hazra and Samanta introduced the smooth topological space which is a generalization of a fuzzy topological space. In [4], we introduced the concept of fuzzy $r$-minimal space which is an extension of the smooth topological space. The concepts of fuzzy $r$-open sets and fuzzy $r-M$ continuous mappings are also introduced and studied.

In this paper, we introduce the concept of fuzzy $r$ -minimal preopen sets and study some related properties. We also introduce the concept of fuzzy $r-M$ precontinuous mappings, which is a generalization of fuzzy $r-M$ continuous mapping. In particular, we investigate some characterization for the fuzzy $r-M$ precontinuous mapping in terms of fuzzy $r$-minimal interior and fuzzy $r$-minimal closure operators.

\section{Preliminaries}

Let $I$ be the unit interval $[0,1]$ of the real line. A member $A$ of $I^{X}$ is called a fuzzy set [5] of $X$. By $\tilde{0}$ and $\tilde{1}$, we denote constant maps on $\mathrm{X}$ with value 0 and 1 , respectively.

접수일자 : 2010년 4월 14일

완료일자 : 2010년 5월 20일
For any $A \in I^{X}, A^{c}$ denotes the complement $\tilde{1}-A$. All other notations are standard notations of fuzzy set theory.

A fuzzy point $x_{\alpha}$ in $X$ is a fuzzy set $x_{\alpha}$ is defined as follows

$$
x_{\alpha}(y)= \begin{cases}\alpha, & \text { if } y=x \\ 0, & \text { if } y \neq x\end{cases}
$$

A fuzzy point $x_{\alpha}$ is said to belong to a fuzzy set $A$ in $X$, denoted by $x_{\alpha} \in A$, if $\alpha \leq A(x)$ for $x \in X$.

A fuzzy set $A$ in $X$ is the union of all fuzzy points which belong to $A$.

Let $f: X \rightarrow Y$ be a mapping and $A \in I^{X}$ and $B \in I^{Y}$. Then $f(A)$ is a fuzzy set in $Y$, defined by

$$
f(A)(y)= \begin{cases}A(z)_{z \in f^{-1}(y)}, & \text { if } f^{-1}(y) \neq \varnothing, \\ 0, & \text { otherwise }\end{cases}
$$

for $y \in Y$ and $f^{-1}(B)$ is a fuzzy set in $X$, defined by $f^{-1}(B)(x)=B(f(x)), x \in X$.

A smooth topology [2, 3] on $X$ is a map $T: I^{X} \rightarrow I$ which satisfies the following properties:

(1) $T(\tilde{0})=T(\tilde{1})=1$.

(2) $T\left(A_{1} \wedge A_{2}\right) \geq T\left(A_{1}\right) \wedge T\left(A_{2}\right)$ for $A_{1}, A_{2} \in I^{X}$.

(3) $T\left(\vee A_{i}\right) \geq \wedge T\left(A_{i}\right)$ for $A_{i} \in I^{X}$.

The pair $(X, T)$ is alled a smooth topological space. 
Let $X$ be a nonempty set and $r \in(0,1]=I_{0}$. A fuzzy family $M: I^{X} \rightarrow I$ on $\mathrm{X}$ is said to have a fuzzy $r$ -minimal structure [4] if the family

$$
M_{r}=\left\{A \in I^{X}: M(A) \geq r\right\}
$$

contains $\tilde{0}$ and $\tilde{1}$.

Then the $(X, M)$ is called a fuzzy $r$-minimal space (simply $r-F M S$ ) [4]. Every member of $M_{r}$ is called a fuzzy $r$-minimal open set. A fuzzy set $A$ is called a fuzzy $r$-minimal closed set if the complement of $A$ (simply, $A^{c}$ ) is a fuzzy $r$-minimal open set.

Let $(X, M)$ be an $r$-FMS and $r \in(0,1]=I_{0}$. The fuzzy $r$-minimal closure of $A$, denoted by $\operatorname{mC}(A, r)$, is defined as

$$
\operatorname{mC}(A, r)=\cap\left\{B \in I^{X:} \quad B^{c} \in M_{r} \text { and } A \subseteq B\right\}[4] .
$$

The fuzzy $r$-minimal interior of $A$, denoted by $\operatorname{mI}(A$, $r)$, is defined as

$$
\mathrm{mI}(A, r)=\cup\left\{B \in I^{X}: \quad B \in M_{r} \text { and } B \subseteq A\right\}[4] .
$$

Theorem 2.1 ([4]). Let $(X, M)$ be an $r-F M S$ and $A$, $B \in I^{X}$. Then the following properties hold:

(1) $\mathrm{mI}(A, r) \subseteq A$ and if $\mathrm{A}$ is a fuzzy $r$-minimal open set, then $\operatorname{mI}(A, r)=A$.

(2) $A \subseteq \mathrm{mC}(A, r)$ and if $A$ is a fuzzy $r$-minimal closed set, then $\operatorname{mC}(A, r)=A$.

(3) If $A \subseteq B$, then $\mathrm{mI}(A, r) \subseteq \mathrm{mI}(B, r)$ and $\mathrm{mC}(A, r) \subseteq$ $\mathrm{mC}(B, r)$.

(4) $\mathrm{mI}(A \cap B, r) \subseteq \mathrm{mI}(A, r) \cap \mathrm{mI}(B, r)$ and $\operatorname{mC}(A, r) \cup$ $\mathrm{mC}(B, r) \subseteq \mathrm{mC}(A \cup B, r)$

(5) $\mathrm{mI}(\mathrm{mI}(A, r), r)=\mathrm{mI}(A, r)$ and $\mathrm{mC}(\mathrm{mC}(A, r), r)=\mathrm{mC}$ $(A, r)$.

(6) $\tilde{1}-\operatorname{mC}(A, r)=\operatorname{mI}(\tilde{1}-A, r)$ and $\tilde{1}-\operatorname{mI}(A, r)=\operatorname{mC}(\tilde{1}-A$, $r)$.

Definition $2.2([4])$. Let $(X, M)$ and $(Y, N)$ be $r$ $-F M S^{\prime}$ S. Then $f: X \rightarrow Y$ is said to be fuzzy $r-M$ continuous function if for every $A \in N_{r}, f^{-1}(A)$ is in $M_{r}$.

Theorem 2.3 ([4]). Let $f: X \rightarrow Y$ be a function on $r$ $-F M S^{\prime} S(X, M)$ and $(Y, N)$.

(1) $f$ is fuzzy $r-M$ continuous

(2) $f^{-1}(B)$ is a fuzzy $r$-minimal closed set, for each fuzzy $r$-minimal closed set $B$ in $Y$.

(3) $f(\mathrm{mC}(A, r)) \subseteq \mathrm{mC}(f(A), r)$ for $A \in I^{X}$.

(4) $\mathrm{mC}\left(f^{-1}(B), r\right) \subseteq f^{-1}(\mathrm{mC}(B, r))$ for $B \in I^{Y}$.

(5) $f^{-1}(\mathrm{mI}(B, r)) \subseteq \mathrm{mI}\left(f^{-1}(B), r\right)$ for $B \in I^{Y}$.

Then $(1) \Leftrightarrow(2) \Rightarrow(3) \Leftrightarrow(4) \Leftrightarrow$ (5).

\section{Fuzzy r-minmal preopen sets and fuzzy $r-M$ precontinuity}

Definition 3.1. Let $(X, M)$ be an $r-F M S$ and $A \in$ $I^{X}$. Then a fuzzy set $A$ is called a fuzzy $r$-minimal preopen set in $X$ if $A \subseteq \mathrm{mI}(\mathrm{mC}(A, r), r)$.

A fuzzy set $A$ is called a fuzzy $r$-minimal spreclosed set if the complement of $A$ is fuzzy $r$-minimal preopen.

Every fuzzy $r$-minimal open set is fuzzy $r$-minimal preopen but the converse may not be true in general.

Example 3.2 Let $X=[0,1]$, let $A, B$ and $C$ be fuzzy sets defined as follows

$$
\begin{array}{ll}
A(x)=\frac{1}{2}(x+1), & x \in I ; \\
B(x)=-\frac{3}{4}(x-1), & x \in I ; \\
C(x)=\frac{1}{5}(x+4), & x \in I .
\end{array}
$$

Let us consider a fuzzy minimal structure $M$ as follows

$$
M(\mu)= \begin{cases}\frac{2}{3}, & \text { if } \mu=\tilde{0}, \tilde{1}, A, B, \\ 0, & \text { otherwise. }\end{cases}
$$

Then the fuzzy set $C$ is fuzzy $\frac{2}{3}$-minimal preopen but not fuzzy $\frac{2}{3}$-minimal open.

Lemma 3.3. Let $(X, M)$ be an $r-F M S$ and $A \in I^{X}$. Then a fuzzy set $A$ is fuzzy $r$-minimal preclosed set if and only if $\operatorname{mC}(\mathrm{mI}(A, r), r) \subseteq A$.

Theorem 3.4. Let $(X, M)$ be an $r-F M S$. Any union of fuzzy $r$-minimal preopen sets is fuzzy $r$-minimal preopen.

Proof. Let $A_{i}$ be a fuzzy $r$-minimal preopen set for $i$ $\in J$. Then from Theorem 2.1, $A_{i} \subseteq \operatorname{mI}\left(\operatorname{mC}\left(A_{i}, r\right), r\right) \subseteq$ $\mathrm{mI}\left(\mathrm{mC}\left(\cup A_{i}, r\right), r\right)$. This implies $\cup A_{i} \subseteq \mathrm{mI}\left(\mathrm{mC}\left(\cup A_{i}, r\right)\right.$, $r$ ) and so $\cup A_{i}$ is fuzzy $r$-minimal preopen.

As shown in the next example, in general, the intersection of two fuzzy $r$-minimal preopen sets may not be fuzzy $r$-minimal preopen.

Example 3.5. Let $X=[0,1]$, let $C, D, E$ and $F$, be fuzzy sets defined as follows

$$
C(x)=\frac{1}{3} x+\frac{1}{2}, \quad \text { if } x \in I
$$




$$
\begin{aligned}
& D(x)=-\frac{1}{3}(x-1)+\frac{1}{2}, \quad \text { if } x \in I \text {; } \\
& E(x)= \begin{cases}-x+\frac{1}{2}, & \text { if } 0 \leq x \leq \frac{1}{4}, \\
\frac{1}{3}(x-1)+\frac{1}{2}, & \text { if } \frac{1}{4} \leq x \leq 1\end{cases} \\
& F(x)= \begin{cases}\frac{1}{3} x+\frac{1}{2}, & \text { if } 0 \leq x \leq \frac{3}{4}, \\
-x+\frac{3}{2}, & \text { if } \frac{3}{4} \leq x \leq 1 .\end{cases}
\end{aligned}
$$

Let us consider a fuzzy minimal structure

$$
N(\mu)= \begin{cases}\frac{2}{3}, & \text { if } \mu=\tilde{0}, \tilde{1}, E, F, \\ 0, & \text { otherwise. }\end{cases}
$$

Then the fuzzy sets $C$ and $D$ are fuzzy $\frac{2}{3}$-minimal preopen. But $C \cap D$ is not fuzzy $\frac{2}{3}$-minimal preopen, because of $\operatorname{mI}\left(\operatorname{mC}\left(C \cap D, \frac{2}{3}\right), \frac{2}{3}\right)=E \cup F \subseteq C, D$ but $E$ $\cup F \neq C, D$.

Definition 3.6. Let $(X, M)$ be an $r-F M S$ and $A \in$ $I^{X}, \operatorname{mpC}(A, r)$ and $\operatorname{mpI}(A, r)$, respectively, are defined as the following:

$$
\begin{aligned}
& \operatorname{mpC}(A, r)=\left\{\left\{\in \in I^{X}: A \subseteq F \text { and } F\right.\right. \text { is fuzzy } \\
&r \text {-minimal preclosed }\}, \\
& \operatorname{mpI}(A, r)=U\left\{U \in I^{X}: \quad U \subseteq A \text { and } U\right. \text { is fuzzy } \\
&r \text {-minimal preopen }\} .
\end{aligned}
$$

Theorem 3.7. Let $(X, M)$ be an $r-F M S$ and $A \in I^{X}$. Then

(1) $\operatorname{mpI}(A, r) \subseteq A$.

(2) If $A \subseteq B$, then $\operatorname{mpI}(A, r) \subseteq \operatorname{mpI}(B, r)$.

(3) $A$ is fuzzy $r$-minimal preopen if and only if $\mathrm{mpI}$ $(A, r)=A$.

(4) $\operatorname{mpI}(\operatorname{mpI}(A, r), r)=\operatorname{mpI}(A, r)$.

(5) $\operatorname{mpC}(\tilde{1}-A, r)=\tilde{1}-\operatorname{mpI}(A, r)$ and $\operatorname{mpI}(\tilde{1}-A, r)=\tilde{1}$ $-\operatorname{mpC}(A, r)$.

Proof. (1), (2), (3) and (4) are obviously obtained from Theorem 3.4.

(5) For $A \in I^{X}$,

$\tilde{1}-\operatorname{mpI}(A, r)$

$=\tilde{1}-\cup\left\{U \in I^{X}: U \subseteq A\right.$ and $U$ is fuzzy $r$-minimal preopen $\}$

$=\cap\{\tilde{1}-U: U \subseteq A, \quad U$ is fuzzy $r$-minimal preopen $\}$
$=\cap\{\tilde{1}-U: \tilde{1}-A \subseteq \tilde{1}-U, \quad U$ is fuzzy $r-$ minimal preopen\}

$=\operatorname{mpC}(\tilde{1}-A, r)$.

Similarly, $\operatorname{mpI}(\tilde{1}-A, r)=\tilde{1}-\operatorname{mpC}(A, r)$.

Theorem 3.8. Let $(X, M)$ be an $r-F M S$ and $A \in I^{X}$. Then

(1) $A \subseteq \operatorname{mpC}(A, r)$.

(2) If $A \subseteq B$, then $\operatorname{mpC}(A, r) \subseteq \operatorname{mpC}(B, r)$.

(3) $F$ is fuzzy $r$-minimal preclosed if and only if $\operatorname{mpC}(F)=F$.

(4) $\operatorname{mpC}(\operatorname{mpC}(A, r), r)=\operatorname{mpC}(A, r)$.

Proof. It is obvious from Theorem 3.7.

Lemma 3.9. Let $(X, M)$ be an $r-F M S$ and $A \in I^{X}$. Then

(1) $x_{\alpha} \in \operatorname{mpC}(A, r)$ if and only if $A \cap V \neq \varnothing$ for $\mathrm{ev}^{-}$

ery fuzzy $r$-minimal preopen set $\mathrm{V}$ containing $x_{\alpha}$.

(2) $x_{\alpha} \in \operatorname{mpI}(A, r)$ if and only if there exists a fuzzy $r$-minimal preopen set $G$ such that $G \subseteq A$.

Proof. (1) If there is a fuzzy $r$-minimal preopen set $V$ containing $x_{\alpha}$ such that $A \cap V=\varnothing$, then $\tilde{1}-V$ is a fuzzy $r$-minimal preclosed set such that $A \subseteq \tilde{1}-V, x_{\alpha}$ $\notin \tilde{1}-V$. So $x_{\alpha} \notin \operatorname{mpC}(A, r)$.

The other relation is obvious.

(2) Obvious

Definition 3.10. Let $(X, M)$ and $(Y, N)$ be $r-F M S$ 's. Then $f: X \rightarrow Y$ is said to be fuzzy $r-M$ preicontinuous if for each fuzzy point $x_{\alpha}$ and each fuzzy $r$-minimal open set $V$ containing $f\left(x_{\alpha}\right)$, there exists a fuzzy $r$ - minimal preopen set $U$ containing $x_{\alpha}$ such that $f(U)$ $\subseteq V$.

Every fuzzy $r-M$ continuous mapping is fuzzy $r-M$ precontinuous but the converse is not true in general.

Example 3.11. Let $X=[0,1]$. Consider two fuzzy minimal structures $(X, M)$ and $(X, N)$ defined in Example 3.2 and Example 3.5, respectively. Then the identity mapping $f:(X, N) \rightarrow(X, M)$ is fuzzy $\frac{2}{3}-M$ precontinuous but not fuzzy $\frac{2}{3}-M$ continuous.

Theorem 3.12. Let $f: X \rightarrow Y$ be a mapping on fuzzy $r$-minimal spaces $(X, M)$ and $(Y, N)$. Then the following are equivalent:

(1) $f$ is fuzzy $r-M$ precontinuous.

(2) $f^{-1}(V)$ is a fuzzy $r$-minimal precopen set for each fuzzy $r$-minimal open set $V$ in $Y$. 
(3) $f^{-1}(B)$ is a fuzzy $r$-minimal precclosed set for each fuzzy $r$-minimal closed set $B$ in $Y$.

(4) $f(\operatorname{mpC}(A, r)) \subseteq \operatorname{mC}(f(A), r)$ for $A \in I^{X}$.

(5) $\operatorname{mpC}\left(f^{-1}(B), r\right) \subseteq f^{-1}(\mathrm{mC}(B, r))$ for $B \in I^{Y}$.

(6) $f^{-1}(\mathrm{mI}(B, r)) \subseteq \operatorname{mpI}\left(f^{-1}(B), r\right)$ for $B \in I^{Y}$.

Proof. (1) $\Rightarrow$ (2) Let $V$ be any fuzzy $r$-minimal open set in $Y$ and $x_{\alpha} \in f^{-1}(V)$. Then there exists a fuzzy $r$ -minimal precopen set $U$ containing $x_{\alpha}$ such that $f(U)$ $\subseteq V$ and so $U \subseteq f^{-1}(V)$ for all $x_{\alpha} \in f^{-1}(V)$. Hence from Theorem 3.4, $f^{-1}(V)$ is fuzzy $r$-minimal precopen.

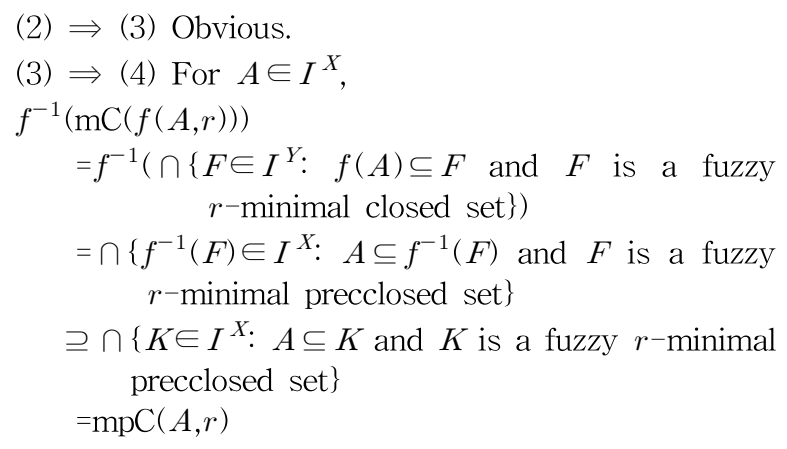

Therefore, $f(\operatorname{mpC}(A, r)) \subseteq \mathrm{mC}(f(A), r)$.

(4) $\Rightarrow$ (5) For $B \in I^{Y}$,

$f\left(\operatorname{mpC}\left(f^{-1}(B), r\right)\right) \subseteq \operatorname{mC}\left(f\left(f^{-1}(B)\right), r\right) \subseteq \mathrm{mC}(B, r)$.

Thus this implies $\operatorname{mpC}\left(f^{-1}(B), r\right) \subseteq f^{-1}(\operatorname{mC}(B, r))$.

(5) $\Rightarrow$ (6) For $B \in I^{Y}$, from Theorem 3.7,

$$
\begin{aligned}
f^{-1}(\operatorname{mI}(B, r))= & f^{-1}(\tilde{1}-\operatorname{mC}(\tilde{1}-B, r)) \\
& =\tilde{1}-\left(f^{-1}(\operatorname{mC}(\tilde{1}-B), r)\right) \\
& \subseteq \tilde{1}-\operatorname{mpC}\left(f^{-1}(\tilde{1}-B)\right) \\
& =\operatorname{mpI}\left(f^{-1}(B), r\right) .
\end{aligned}
$$

Hence $f^{-1}(\operatorname{mI}(B, r)) \subseteq \operatorname{mpI}\left(f^{-1}(B), r\right)$.

(6) $\Rightarrow$ (1) Let $V$ be any fuzzy $r$-minimal open set containing $f\left(x_{\alpha}\right)$ for a fuzzy point $x_{\alpha}$. From (6), it follows

$$
x_{\alpha} \in f^{-1}(V)=f^{-1}(\mathrm{mI}(V, r)) \subseteq \operatorname{mpI}\left(f^{-1}(V), r\right) .
$$

Since $x_{\alpha} \in \operatorname{mpI}\left(f^{-1}(V), r\right)$, there exists a fuzzy $r$ -minimal precopen set $U$ containing $x_{\alpha}$ such that $U \subseteq$ $f^{-1}(V)$. Hence $f$ is fuzzy $r-M$ preccontinuous.

Lemma 3.13. Let $(X, M)$ be an $r-F M S$ and $A \in I^{X}$. Then

(1) $\mathrm{mC}(\mathrm{mI}(A, r), r) \subseteq \mathrm{mC}(\mathrm{mI}(\operatorname{mpC}(A, r), r), r) \subseteq \operatorname{mpC}(A, r)$.

(2) $\operatorname{mpI}(A, r) \subseteq \mathrm{mI}(\mathrm{mC}(\operatorname{mpI}(A, r), r), r) \subseteq \mathrm{mI}(\operatorname{mC}(A, r), r)$.

Proof. (1) Since $A \subseteq \operatorname{mpC}(A, r)$ and $\operatorname{mpC}(A, r)$ is fuzzy $r$-minimal preclosed, it is obtained from Lemma 3.3 and Theorem 3.8 .
(2) Similarly, it follows from Theorem 3.7.

Theorem 3.14. Let $f: X \rightarrow Y$ be a mapping on $r$ -FMS's $(X, M)$ and $(Y, N)$. Then the following statements are equivalent:

(1) $f$ is fuzzy $r-M$ precontinuous.

(2) $f^{-1}(V) \subseteq \mathrm{mI}\left(\mathrm{mC}\left(f^{-1}(V), r\right), r\right)$ for each fuzzy $r$ -minimal open set $V$ in $Y$.

(3) $\mathrm{mC}\left(\mathrm{mI}\left(f^{-1}(F), r\right), r\right) \subseteq f^{-1}(F)$ for each fuzzy $r$ -minimal closed set $F$ in $Y$.

(4) $f(\mathrm{mC}(\mathrm{mI}(A, r), r)) \subseteq \mathrm{mC}(f(A), r)$ for $A \in I^{X}$.

(5) $\mathrm{mC}\left(\mathrm{mI}\left(f^{-1}(B), r\right), r\right) \subseteq f^{-1}(\mathrm{mC}(B, \mathrm{r}))$ for $B \in I^{Y}$.

(6) $f^{-1}(\mathrm{mI}(B, r)) \subseteq \mathrm{mI}\left(\mathrm{mC}\left(f^{-1}(B), r\right), r\right)$ for $B \in I^{Y}$.

Proof. (1) $\Leftrightarrow$ (2) It is obtained from definition of fuzzy $r$-minimal preopen sets and Theorem 3.12.

(1) $\Leftrightarrow$ (3) It follows from Lemma 3.3 and Theorem 3.12 .

(3) $\Rightarrow$ (4) Let $A \in I^{X}$. Then from Lemma 3.13(1) and hypothesis, it follows $\mathrm{mC}(\mathrm{mI}(A, r), r) \subseteq \operatorname{mpC}(A, r)$ $\subseteq f^{-1}(f(\operatorname{mpC}(A, r))) \subseteq f^{-1}(\mathrm{mC}(f(A), r))$.

Hence $f(\operatorname{mC}(\operatorname{mI}(A, r), r)) \subseteq \operatorname{mC}(f(A), r)$.

(4) $\Rightarrow$ (5) Obvious.

(5) $\Rightarrow$ (6) Let $B \in I^{Y}$. Then from Theorem 2.1,

$f^{-1}(\mathrm{mI}(B, r))=f^{-1}(\tilde{1}-\mathrm{mC}(\tilde{1}-B, r))$ $=\tilde{1}-\left(f^{-1}(\mathrm{mC}(\tilde{1}-B, r))\right)$

$\subseteq \tilde{1}-\mathrm{mC}\left(\mathrm{mI}\left(f^{-1}(\tilde{1}-B), r\right), r\right)$ $=\mathrm{mI}\left(\mathrm{mC}\left(f^{-1}(B), r\right), r\right)$.

Hence (6) is obtained.

(6) $\Rightarrow$ (1) Let $V$ be any fuzzy $r$-minimal open set. Then since $V=\mathrm{mI}(V, r)$, by (6), we have $f^{-1}(V)=f^{-1}$ $(\mathrm{mI}(V, r)) \subseteq \mathrm{mI}\left(\mathrm{mC}\left(f^{-1}(V), r\right), r\right)$. By Theorem 3.7 (3), $f^{-1}(V)$ is fuzzy $r$-minimal preopen and so $f$ is fuzzy $r$ $-M$ precontinuous.

\section{References}

[1] C L. Chang, "Fuzzy topological spaces", J. Math. Anal. Appl., vol. 24, pp. 182-190, 1968.

[2] K. C. Chattopadhyay, R. N. Hazra, and S. K. Samanta, "Gradation of openness: Fuzzy topology", Fuzzy Sets and Systems, vol. 49, pp. 237-242, 1992.

[3] A. A. Ramadan, "Smooth topological spaces", Fuzzy Sets and Systems, vol. 48, pp. 371-375, 1992.

[4] Y. H. Yoo, W. K. Min and J. I. Kim. "Fuzzy $r$ -Minimal Structures and Fuzzy $r$-Minimal Spaces", Far East Journal of Mathematical Sciences, vol. 33, no. 2, pp.193-205, 2009. 
Fuzzy $r$-minimal Preopen Sets And Fuzzy $r-M$ Precontinuous Mappings On Fuzzy Minimal Spaces

[5] L. A. Zadeh, "Fuzzy sets", Information and Control, pp. 338-353, 1965.

저 자 소 개

Won Keun Min

Department of Mathematics, Kangwon National

University, Chuncheon, 200-701, Korea

wkmin@kangwon.ac.kr

Young Key Kim

Department of Mathematics, MyongJi University,

Youngin 449-728, Korea.

ykkim@mju.ac.kr 